\title{
Symmetry of Tangent Stiffness Matrices of 3D Elastic Frame ${ }^{a}$
}

\author{
B.A. Izzuddin ${ }^{2}$
}

In their paper, the authors contend to have resolved the issues concerning the symmetry of the tangent stiffness matrix for spatial elastic beam elements and frames, suggesting that the element tangent stiffness matrix is invariably asymmetric. Furthermore, they suggest that an asymmetric geometric stiffness 'correction' matrix is required for the detection of the lowest buckling mode of a space dome, citing the failure of several other researchers, including the discusser (Izzuddin and Elnashai, 1993), to detect this mode. The discusser has two contributions to make in connection with the aforementioned claims of the authors.

\section{Asymmetry of Element Tangent Stiffness Matrices}

In order to generalise regarding the symmetry of the tangent stiffness matrix of spatial elastic beam elements and frames, an unambiguous definition of the 'scope' of the tangent stiffness matrix is required, which is not provided by the authors. This particularly concerns the nature of 'applied' loading (particularly moments) and system freedoms (particularly rotations) which the tangent stiffness matrix is associated with. More clearly, the system tangent stiffness matrix (K) can be considered to reflect the infinitesimal variation of out-of-balance forces and moments $(\mathrm{G})$ with system translational and rotational freedoms $(\mathrm{U})$, as expressed by:

$$
K_{i, j}=\frac{\partial G_{i}}{\partial U_{j}}
$$

\footnotetext{
${ }^{a}$ February, 1999, Journal of Engineering Mechanics, Vol. 125, No.2, by L.H. Teh and M.J. Clarke (Technical Note No. 14141)

${ }^{2}$ Lecturer in Engineering Computing, Department of Civil and Environmental Engineering, Imperial College, London SW7 2BU, U.K.
} 
Adopting a variational formulation, $\mathrm{G}$ can be related to the first derivative of the total potential energy ( $\Pi)$ of the system (which is the sum of the system strain energy (U) and the load potential energy $(-\mathrm{W}))$ with respect to $\mathrm{U}$ :

$$
\mathrm{G}_{\mathrm{i}}=\frac{\partial \Pi}{\partial \mathrm{U}_{\mathrm{i}}}=\frac{\partial \mathrm{U}}{\partial \mathrm{U}_{\mathrm{i}}}-\frac{\partial \mathrm{W}}{\partial \mathrm{U}_{\mathrm{i}}}=\mathrm{R}_{\mathrm{i}}-\mathrm{P}_{\mathrm{i}}^{\mathrm{e}}
$$

In the above, $R$ represents the resistance forces (which are work conjugate with $U$ ) and $P^{e}$ represents 'equivalent' nodal loading. $P^{e}$ is identical to the actual applied loading $(P)$ if the latter is work conjugate with $\mathrm{U}$ (i.e. $\mathrm{W}=\mathrm{P}^{\mathrm{T}} \mathrm{U}$ ), but otherwise $\mathrm{P}^{\mathrm{e}}$ would be a function of $\mathrm{P}$ and possibly $U$ ( $P$ is taken to be independent of $U)$.

An alternative approach is to re-arrange (2) so that $G$ is defined as the difference between 'equivalent' resistance forces $\left(R^{\mathrm{e}}\right)$ and the actual applied nodal loading $(P)$ :

$$
\mathrm{G}_{\mathrm{i}}=\mathrm{R}_{\mathrm{i}}^{\mathrm{e}}-\mathrm{P}_{\mathrm{i}}
$$

In both approaches, $\mathrm{G}$ can be considered to represent the errors in the equilibrium equations.

Clearly, the use of the former approach represented by (2) always results in a symmetric tangent stiffness matrix, provided that the elastic system is subjected to conservative loading (leading to a continuous $\Pi$ which is uniquely defined in terms of $U$ ), since:

$$
K_{i, j}=\frac{\partial^{2} \Pi}{\partial U_{i} \partial U_{j}}=\frac{\partial^{2} \Pi}{\partial U_{j} \partial U_{i}}=K_{j, i}
$$

If $P$ is not work conjugate with $U$, the two approaches (2) and (3) result in different equilibrium equations, and the tangent stiffness matrix required for the latter approach according to (1) may be asymmetric. However, it must be emphasised that both approaches lead to identical solutions if the same deformation modes and strain-displacement relationships are employed, and hence it is always possible to achieve a symmetric tangent stiffness matrix for a conservative elastic system by adopting the first approach. 
Accordingly, the discusser proposes that asymmetry in the tangent stiffness matrix arises only with the second approach and only if the applied loading $(P)$ is not work conjugate with the system freedoms $(\bigcup)$. Therefore, in the context of spatial elastic frames, asymmetry of the tangent stiffness matrix, both on the element and overall system levels, arises only with the second approach and only if the 'applied' moments in $\mathrm{P}$ are not work conjugate with the rotational freedoms in $U$ (assuming that the 'applied' forces in $\mathrm{P}$ are work conjugate with the translational freedoms in $U$ ).

In his work on spatial frames (Izzuddin, 1991; Izzuddin and Elnashai, 1993), the discusser adopted the first approach, formulating the element strain energy in a convected (corotational) framework, and achieving a symmetric tangent stiffness matrix both on the element and system levels. The discusser simplified this approach further by assuming that all 'applied' moments in $\mathrm{P}$ are zero, thus leading to considerable simplification in determining $\mathrm{P}^{\mathrm{e}}$, where the equivalent nodal moments in $\mathrm{P}^{\mathrm{e}}$ become also zero (Izzuddin and Elnashai, 1993). Consequently, the nature of the resistance moments in $R$ become only of academic interest, although the discusser showed that, for the adopted definition of rotational freedoms in $U$, the moments in $R$ are of the semi-tangential type but considered in an incremental context (Izzuddin and Elnashai, 1993). Since most realistic spatial framed structures are not subjected to directly 'applied' moments, and since, in any case, most conservative 'applied' moments can be represented by conservative forces acting on rigid links, the aforementioned simplification is justified.

The discusser disagrees with authors on the significance of the nature of 'internal' bending moments for the symmetry of the element and system tangent stiffness matrix. Only the nature of 'applied' moments in relation to the assumed definition of rotational freedoms is relevant in this respect, and only if the variational approach in (2) is not adopted. The discusser contends that 'internal' bending moments should only be considered as generalised stresses which are work conjugate with curvature generalised strains, and that the assertion by the authors that 'internal' bending moments are work conjugate with vectorial rotations is misleading. The accusation by the authors of "many other researchers ..." of "... ignorance of 
the fact that bending moments are work conjugate with vectorial rotations, not with transverse displacement derivatives." is ill-founded. In fact, the discusser showed that the assumption of work conjugacy between bending moments and the second derivatives of transverse displacements (approximating curvatures) is fully justified if a convected (corotational) framework is adopted for the formulation of the element strain energy and a sufficient number of elements is used per member (Izzuddin and Lloyd Smith, 1996). The accuracy of this assumption and the success of the discusser's approach is further illustrated with the space dome example used by the authors.

\section{Space Dome Example}

The authors suggest that an asymmetric 'correction' geometric stiffness matrix is necessary to predict the lowest buckling mode of a space dome structure. They also imply that many other researchers, including the discusser (Izzuddin and Elnashai, 1993), could not detect this mode due to a serious "ignorance", as mentioned previously. While, in their nonlinear analysis, the authors employed a specific technique for detecting bifurcation equilibrium states, the discusser undertook the nonlinear analysis on a perfect dome without employing such a technique, which explains the previous results of the discusser. In order to illustrate the accuracy of the discusser's method (Izzuddin, 1991; Izzuddin and Elnashai, 1993), which does not require an asymmetric 'correction' matrix, the nonlinear analysis is performed here on an imperfect dome, and the results are shown alongside the predictions from the previous perfect dome in Figure A. Random imperfections are introduced to the nodal positions, which vary between 0.001 and 0.003 units, thus enabling a close approximation of the secondary equilibrium path without the need for a bifurcation detection technique. The nonlinear analysis is undertaken with one and two elastic quartic elements (Izzuddin, 1991; Izzuddin, 1996) per member, respectively, where an excellent comparison is obtained between the two sets of results for both the prefect and imperfect space domes. With only one element per member, the discusser's method provides a good comparison against the authors' predictions for both the primary and secondary equilibrium paths, thus illustrating the accuracy of the 
discusser's method, which is completely based on symmetric tangent stiffness matrices for the element and for the overall system.

\section{Appendix: References}

Izzuddin, B.A., 1991, "Nonlinear Dynamic Analysis of Framed Structures", PhD Thesis, Department of Civil Engineering, Imperial College, University of London.

Izzuddin, B.A., and Elnashai, A.S., 1993, "Eulerian Formulation for Large Displacement Analysis of Space Frames", Journal of Engineering Mechanics, ASCE, Vol. 119, No. 3, pp. 549-569.

Izzuddin, B.A., and Lloyd Smith, D., 1996, "Large Displacement Analysis of Elastoplastic Thin-Walled Frames. I: Formulation and Implementation”, Journal of Structural Engineering, ASCE, Vol. 122, No. 8, pp. 905-914.

Izzuddin, B.A., 1996, "Quartic Formulation for Elastic Beam-Columns Subject to Thermal Effects”, Journal of Engineering Mechanics, ASCE, Vol. 122, No. 9, pp. 861-871. 


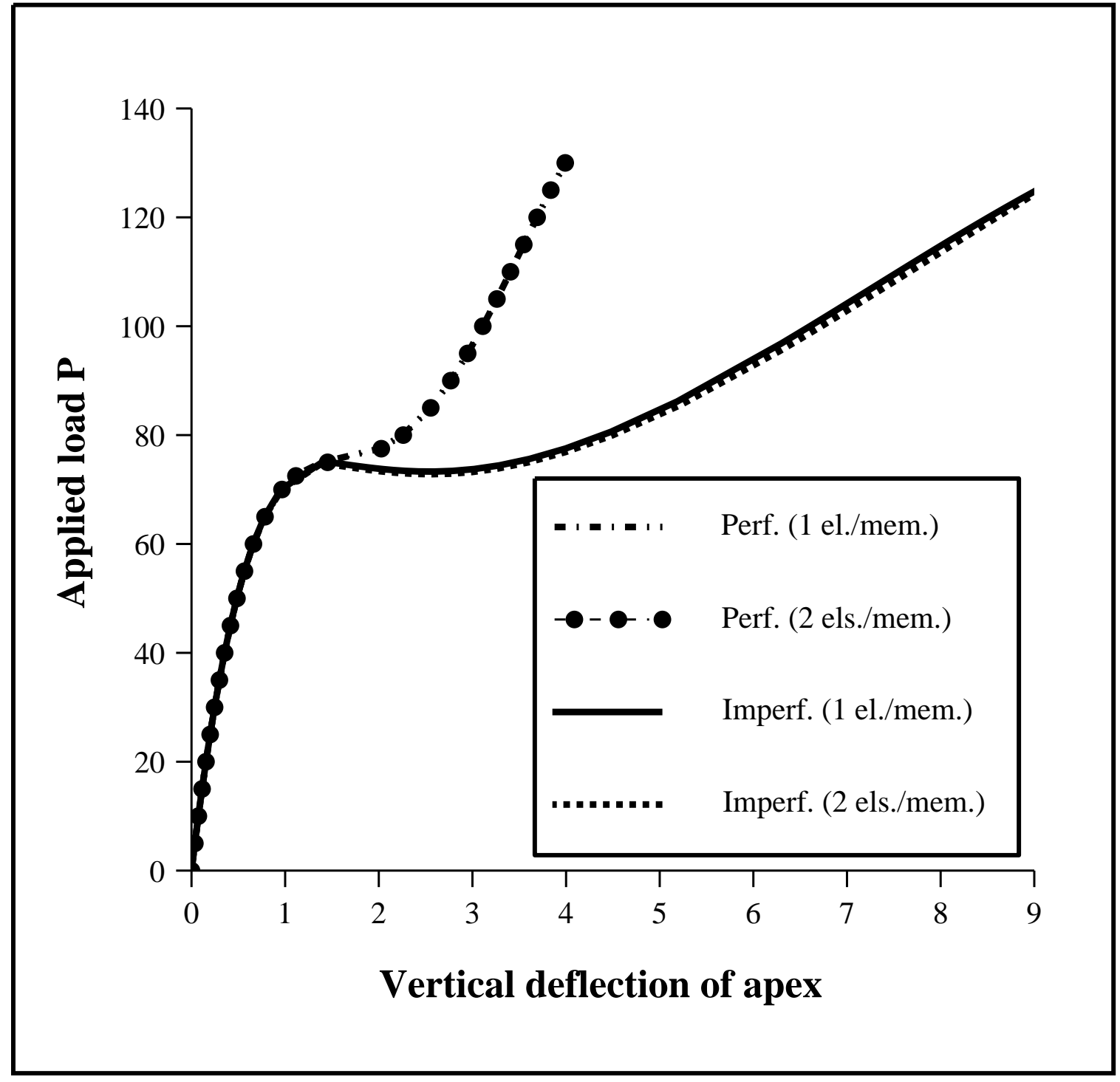

Figure A. Nonlinear response of perfect and imperfect space dome 